Revue internationale P.M.E.

Économie et gestion de la petite et moyenne entreprise

\title{
Évaluer la formation dans des PME françaises : résultats d'une étude empirique
}

\section{Agnès Paradas}

Volume 11, numéro 2-3, 1998

URI : https://id.erudit.org/iderudit/1009042ar

DOI : https://doi.org/10.7202/1009042ar

Aller au sommaire du numéro

Éditeur(s)

Presses de l'Université du Québec

ISSN

0776-5436 (imprimé)

1918-9699 (numérique)

Découvrir la revue

Citer cet article

Paradas, A. (1998). Évaluer la formation dans des PME françaises : résultats d'une étude empirique. Revue internationale P.M.E., 11(2-3), 31-48.

https://doi.org/10.7202/1009042ar
Résumé de l'article

La formation professionnelle connaît un développement important dans les PME françaises, mais ne sera reconnue parles professionnels qu'à condition de pouvoir en évaluer les effets sur la performance de l'organisation.

L'exploitation des résultats d'une enquête menée auprès de 180 PME du Sud de la France, au moyen d'une analyse factorielle des correspondances multiples, permet une réflexion sur les relations de causalité entre les différentes politiques de formation et la performance de la PME.

Si les PME ayant une politique de formation plus élaborée semblent être également les plus performantes, il convient toutefois de s'interroger sur les caractéristiques générales des PME de l'échantillon. 


\title{
Évaluer la formation dans des PME françaises: résultats d'une étude empirique
}

Agnès PARADAS

Institut universitaire technologique

\author{
MOTS CLÉS
}

\section{PME françaises - Formation professionnelle Analyse factorielle des correspondances - Déterminants de la performance}

\begin{abstract}
RÉSUMÉ
La formation professionnelle connaît un développement important dans les PME françaises, mais ne sera reconnue par les professionnels qu'à condition de pouvoir en évaluer les effets sur la performance de l'organisation.

L'exploitation des résultats d'une enquête menée auprès de 180 PME du Sud de la France, au moyen d'une analyse factorielle des correspondances multiples, permet une réflexion sur les relations de causalité entre les différentes politiques de formation et la performance de la PME.

Si les PME ayant une politique de formation plus élaborée semblent être également les plus performantes, il convient toutefois de s'interroger sur les caractéristiques générales des PME de l'échantillon.
\end{abstract}

\section{L'AUTEURE}

Agnès Paradas est maître de conférences à l'Institut universitaire technologique (IUT) de Valence, lequel dépend de l'Université Pierre-Mendès-France à Grenoble, titulaire d'un doctorat en sciences de gestion et membre de l'ERFI (Équipe de recherche sur la girme et l'industrie) à l'Université de Montpellier I.

Ses recherches portent principalement sur les aspects de gestion des ressources humaines (en particulier formation et recrutement) dans les petites entreprises, et sur les liens existant entre les dirigeants - leur profil, leur histoire - et leur mode de gestion de l'entreprise.

Adresse: 13520, Les Baux de Provence, France, Téléphone et télécopieur : 04-90-5452-77, aparadas@ club-internet.fr et IUT TC, BP 29, 26901 Valence Cedex 9, France, Courriel : agnès.paradas@iut-valence.fr 


\section{ABSTRACT}

Professional training in French businesses is very developed and requires an evaluation to have a real meaning for managers. This study presents an empirical research (180 small businesses in South of France), with factorial analysis, and purposes an observation of relations between training policy and performance.

Businesses which have a "good" training seem to be more performed, but other determinant material must be identified.

\section{RESUMEN}

La formación profesional conoce un desarrollo importante en las pequeñas empresas francesas, pero no será reconocida por los profesionales hasta que su incidencia sobre la performancia de la organización sea probada.

La explotación de los resultados de una encuesta llevada a cabo en 180 PyMEs de sur de Francia, por medio del análisis factorial de correspondencias, permite una reflexión sobre las relaciones de causalidad entre las diferentes políticas de formación y la performancia de la PyME.

Si bien las PyMEs que tienen una política de formación más elaborada parecen ser igualmente las más performantes, conviene no obstante interrogarse sobre las características generales de las PyMEs de la muestra.

\section{Introduction}

La formation professionnelle continue revêt en France un caractère obligatoire dans les grandes entreprises comme dans les PME. Celles-ci doivent verser 1,5\% de leur masse salariale au titre de cette formation, soit à des fonds d'assurance formation mutualisateurs (FAF), soit pour leur propre compte, soit au Trésor public comme impôt.

De plus en plus de petites structures, dont les responsables croient à la formation, passent d'une formation «dépense obligatoire » à une formation « investissement » (Caspar et Afriat, 1988 ; Walther, 1988). Intuitivement, la plupart réalisent que certaines formations sont nécessaires, voire indispensables, à la survie de l'entreprise. Transformer cette intuition en certitude permettrait de réduire encore le nombre des PME qui ne forment pas ou qui poursuivent des logiques d'actions ponctuelles (AGEFOS-PME, 1992; CEREQ, 1992).

Les PME apparaissent comme des acteurs de plus en plus importants de notre économie (Beccarelli et Perker, 1991). Éléments fondamentaux du dynamisme régional, elles disposent de peu d'outils pour se repérer dans l'offre de formation, souvent coûteuse, mal adaptée et quelquefois inefficiente. Prouver que la formation apporte des éléments de performance concrets dans l'entreprise permettrait certainement de développer davantage ces actions. 
Sans cela, les responsables risquent d'abandonner la formation, faute de montrer que celle-ci valorise les individus et l'entreprise (Dixon, 1987).

Il s'agira tout d'abord de décrire les variables déterminantes des politiques de formation ainsi que les modèles de référence exploités, afin de pouvoir présenter les objectifs et la méthodologie de l'étude. Les résultats de celle-ci pourront alors être exposés et détaillés dans le but de rester fidèle aux données, de proposer une image correcte des relations existant entre les actions de formation et la performance, et de réfléchir sur d'éventuels rapports de causalité. Ces travaux permettront de prendre du recul par rapport à l'étude et de mener une réflexion sur les logiques de formation et la performance liée de l'entreprise, selon ses caractéristiques.

\section{Cadre d'analyse}

L'AFNOR (1992) définit la formation comme un ensemble mis en œuvre permettant à des personnes sorties du système scolaire d'atteindre un niveau de connaissance et de savoir-faire constituant l'objectif de formation.

Une dimension manque à cette définition, surtout tournée vers l'individu. En effet, la formation perfectionne des qualités humaines particulièrement importantes dans la PME et permet d'améliorer le système de gestion des organisations. Kirkpatrick (1976) propose quatre niveaux d'évaluation de la formation, correspondant à quatre niveaux d'action :

- l'évaluation «à chaud» sur les modalités de la formation suivie ;

- l'évaluation des connaissances des individus;

- l'évaluation du transfert des acquis sur le lieu de travail ;

- l'évaluation des résultats de la formation sur l'entreprise.

Ce quatrième niveau apparaît comme celui qui correspond le mieux aux interrogations d'un chercheur en sciences de gestion (Martory, 1992), la formation étant perçue comme un important moyen d'améliorer la performance de l'entreprise (Caron, 1994). L'enjeu le plus important se situe à ce niveau. Mais les difficultés méthodologiques s'amplifient lorsque l'évaluation touche l'entreprise plutôt que l'individu (Maher et Kur, 1983 ; Kirkpatrick, 1977). De nombreuses variables autres que la formation pourront agir sur le système de gestion et rendre difficile de relier tels résultats à telle ou telle action.

Considérant la configuration classique de la PME (Mintzberg, 1990), les résultats semblent plus directs et moins diffus, ce qui facilite certainement la perception de l'impact de la formation sur le système de gestion (Roger, 1985). Le fait de travailler en PME apporte donc certains avantages méthodologiques et réduit les difficultés rencontrées. 
Pourtant, le monde des PME reste très hétérogène et la formation, dans cet univers, ne constitue pas une réalité unidimensionnelle (Bel et Rosanvallon, 1991; Caron, 1994). Des attitudes et des comportements diversifiés dans la formation doivent pouvoir être identifiés, et donc permettre la mise en évidence de politiques variées.

\subsection{Déterminer les politiques de formation}

La description de ces politiques de formation passe par le repérage d'indicateurs déterminants, décrivant les principales modalités de mise en œuvre. Il convient de présenter ces déterminants.

Les dépenses de formation apparaissent clairement sur les états de suivi de formation en France et permettent essentiellement de mesurer le degré d'implication général de l'entreprise dans la formation. Les PME choisissant de consacrer un pourcentage supérieur à la dépense obligatoire sont plutôt rares, à moins de profiter temporairement des fonds mutualisés des FAF. La proportion d'entreprises dépensant plus que le pourcentage paraît augmenter avec la taille (CEREQ, 1992).

Dans les petites structures, la modalité la plus caractéristique de la formation semble concerner le plan de formation. En effet, il semble que la planification en PME reste peu développée (Van Auken, 1982 ; D’Amboise et Bakanibona, 1990 ; D'Amboise, 1994), cela étant vérifié pour les actions de gestion des ressources humaines, généralement moins formelles dans les plus petites structures (McEvoy, 1983 ; Mahé de Boislandelle, 1994). Il reste possible d'appliquer cette constatation à la planification en formation, souvent limitée (McEvoy, 1983). Pourtant, les plans de formation doivent permettre d'assurer une meilleure cohérence, tout en laissant une certaine souplesse (plans flexibles, semestriels, etc.).

La responsabilité de formation doit également permettre de décrire certaines politiques de formation. La place et le profil des actions se dérouleront différemment selon les gestionnaires de ces actions. Une prise en charge par le chef d'entreprise, par exemple, peut présenter l'avantage d'une meilleure intégration à la stratégie de l'entreprise, avec quelquefois l'inconvénient d'un traitement bâclé. Les entreprises ayant des DRH sont également celles qui considèrent le plus la formation comme un investissement (Tache et Déjean, 1991). Enfin, déléguer la gestion de la formation à un FAF qui assurera une mission de médiateur ou de conseil (Déjean et Willems, 1991) comporte le risque classique de l'externalisation, soit celui de ne pas respecter une cohérence globale entre les différentes actions et le plan d'action général.

Autre déterminant important, le plan engagement-développement représente une mesure gouvernementale destinée à développer la formation dans les PME. Il se concrétise par un contrat que l'entreprise signe avec les institutions légales, 
s'engageant à mettre en œuvre certaines formations moyennant une compensation financière de la part de l'État. Ces plans accroissent la rigueur des politiques de formation.

Une autre modalité essentielle de la politique de formation reste la projection des actions dans le temps. Certains responsables parviennent à élaborer des projets de formation à plus long terme qui sont intégrés à une stratégie générale d'entreprise. D'autres se contentent d'actions ponctuelles « au coup par coup », souvent réactives face à une situation de crise. Une vision à long terme reste davantage liée à une formalisation accrue et à une vision plus proactive, permettant d'anticiper les problèmes. Il semblerait que les efforts de formation engagés dans les PME ne prennent pas assez en considération les projets à long terme de l'entreprise (Fairfield-Sonn, 1987).

Enfin, l'évaluation de la formation, telle qu'elle est pratiquée dans l'entreprise, donne des informations sur la perception de l'importance de la formation et sur les moyens que se donne l'entreprise pour contrôler ses résultats. Souvent informelle, elle peut toutefois apparaître comme réfléchie et explicite. Une politique de formation qui tient une place importante dans l'entreprise sera valorisée par un effort d'évaluation.

\subsection{Approcher la performance}

Afin de pouvoir effectuer une liaison entre action de formation et résultats de l'entreprise, l'approche de la performance semble indispensable, l'évaluation de la réussite pouvant présenter de multiples facettes (Baldwin, 1994).

Le choix d'approcher cette performance de manière globale, systémique et dynamique implique de trouver un modèle général adapté. Le modèle ayant servi de base à la recherche d'indicateurs de performance est celui de Marchesnay (1992) dans lequel on représente le système de gestion de la PME par une schéma articulé autour de quatre pôles principaux : les buts du dirigeant, l'environnement, l'organisation et les activités. Ces pôles sont reliés par des notions clés, telles que le métier, la mission, la culture, l'identité ou le plan d'action de l'entreprise. La performance est appréhendée à travers l'évolution perçue des différents indicateurs, construits à partir d'une revue de littérature traitant des influences possibles de la formation sur les composantes du système de gestion de l'entreprise.

\subsection{Objectifs de recherche et méthodologie}

L'objet du présent article est de recenser les différentes politiques de formation à travers les modalités décrites avant de s'interroger sur leurs influences éventuelles sur le système de gestion. Cet objet peut être traduit par trois interrogations majeures : 
- Existe-t-il différentes politiques de formation dans les PME ?

- Peut-on dégager une liaison positive significative entre certaines de ces politiques et la performance de la PME ?

- Peut-on déduire des rapports de causalité entre ces politiques de formation et la performance?

Pour répondre à ces questions, une enquête a été menée auprès de 212 PME du Sud de la France. Par souci d'homogénéité et de réduction des biais, l'échantillon contient finalement 180 entreprises dont l'effectif reste compris entre 10 et 250 salariés de tous secteurs et niveaux d'activité.

Le questionnaire se compose de deux parties, l'une traitant des évolutions perceptibles dans le système de gestion, l'autre traduisant les actions de formation engagées. Seules des PME faisant un minimum de formation ont été choisies, sans quoi une recherche sur l'évaluation de la formation n'aurait pas eu de sens.

Les questionnaires utilisés regroupent plus de 150 variables et comportent des questions fermées, semi-ouvertes et ouvertes, ce qui donne une certaine souplesse à l'analyse quantitative et permet de ne pas s'enfermer dans des vérités fixées a priori.

Le traitement de ces questionnaires a été réalisé par une analyse factorielle des correspondances multiples, des classifications hiérarchiques ascendantes selon la méthode de Ward, et certains calculs de profils. Les précautions méthodologiques apparaissent nécessaires et doivent être rigoureuses pour donner une valeur à ce type d'analyse.

Le traitement permet alors des combinaisons des variables présentées ci-dessous dans un schéma de lecture générale du questionnaire, rendant possibles la synthèse et la présentation de toutes les données recueillies.

\section{Les résultats}

\subsection{Les différentes politiques de formation dans la PME}

L'analyse porte tout d'abord sur les attitudes des PME au regard de la formation et sur le contenu de leur politique de formation. Les différentes entreprises étudiées ne font pas toutes preuve du même dynamisme ou de la même rigueur dans leurs actions. Pour mieux cerner ces différentes attitudes, 10 variables traduisant cette politique de formation ont été sélectionnées:

- existence d'un plan de formation (fpla);

- cotisation à un fond d'assurance formation (ffaf);

- dépense supérieure au pourcentage obligatoire (fpou); 
SCHÉMA 1

Modèle de base de traitement des données

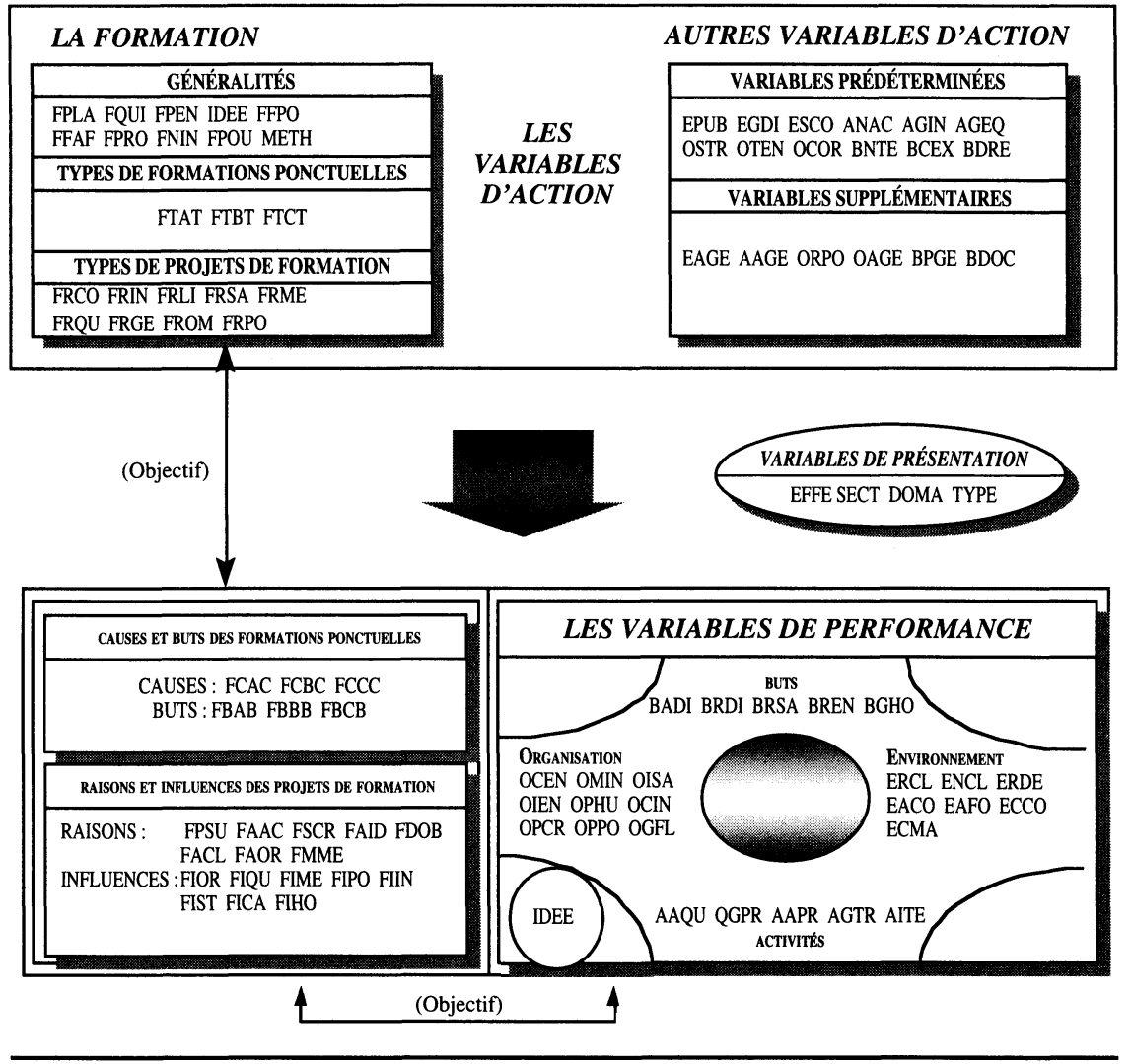

- personne qui gère la formation dans la PME (fqui);

- existence d'un plan engagement-développement (fped);

- mise en place de projets de formation (fpro);

- nombre d'individus concernés par le projet (fnin);

- existence de formations plus ponctuelles (ffpo);

- opinion sur la formation (idee);

- méthode d'évaluation des résultats de la formation (meth).

D'après l'analyse factorielle, les valeurs propres en ordre descendant sont présentées dans le tableau suivant. 
TABLEAU 1

Valeurs propres

\begin{tabular}{lccc}
\hline & Valeurs propres & Trace & \% cumulé \\
\hline 1. & 0,07 & 17,55 & 17,55 \\
2. & 0,06 & 15,72 & 33,27 \\
3. & 0,03 & 8,06 & 41,34 \\
4. & 0,03 & 7,52 & 48,86 \\
5. & 0,02 & 5,87 & 54,73 \\
6. & 0,02 & 5,26 & 59,99 \\
\hline
\end{tabular}

Un grand nombre d'axes explique la position des individus sur le plan. Cependant, on remarque une cession entre les significations des deux premiers axes et les suivants.

Le nombre de variables étant de 10, la comparaison du rapport 1/10 et des valeurs propres indique qu'aucun axe n'a une valeur propre supérieure à 0,1 , bien que les deux premiers s'en rapprochent.

De plus, une vérification par la classification hiérarchique ascendante, menée sur les deux premiers axes, puis sur les six premiers, permet d'affirmer que la tendance semble largement définie par le premier plan factoriel.

Ce constat incite donc à ne conserver pour cette analyse que les deux premiers axes.

Dans le plan factoriel constitué de ces deux premiers axes, l'analyse factorielle des correspondances multiples permet de dégager trois classes.

Le premier axe oppose les PME fortement engagées dans la formation (à droite) et celles qui le sont peu (à gauche).

Le second axe marque une opposition entre des entreprises faisant de la formation de manière ponctuelle et déléguée (en bas) et celles qui n'ont pas donné leur opinion (en haut).

Les trois partitions dégagées apparaissent nettement sur le schéma 2.

La première classe correspond à des PME dont les responsables n'ont pas répondu aux questions posées ou qui n'avaient pas les informations nécessaires pour le faire. Ils sont nommés les «indifférents ».

La deuxième classe, celle des "passionnés », est constituée d'une majorité de réponses positives aux différentes questions, traduisant une démarche volontaire, régulière et active en matière de formation. La plupart de ces entreprises n'adhèrent pas à un FAF, et elles engagent peu de formation ponctuelle. Par contre, elles affirment avoir un plan de formation, des projets, un plan engagement-développement depuis 
SCHÉMA 2

Représentation du plan factoriel

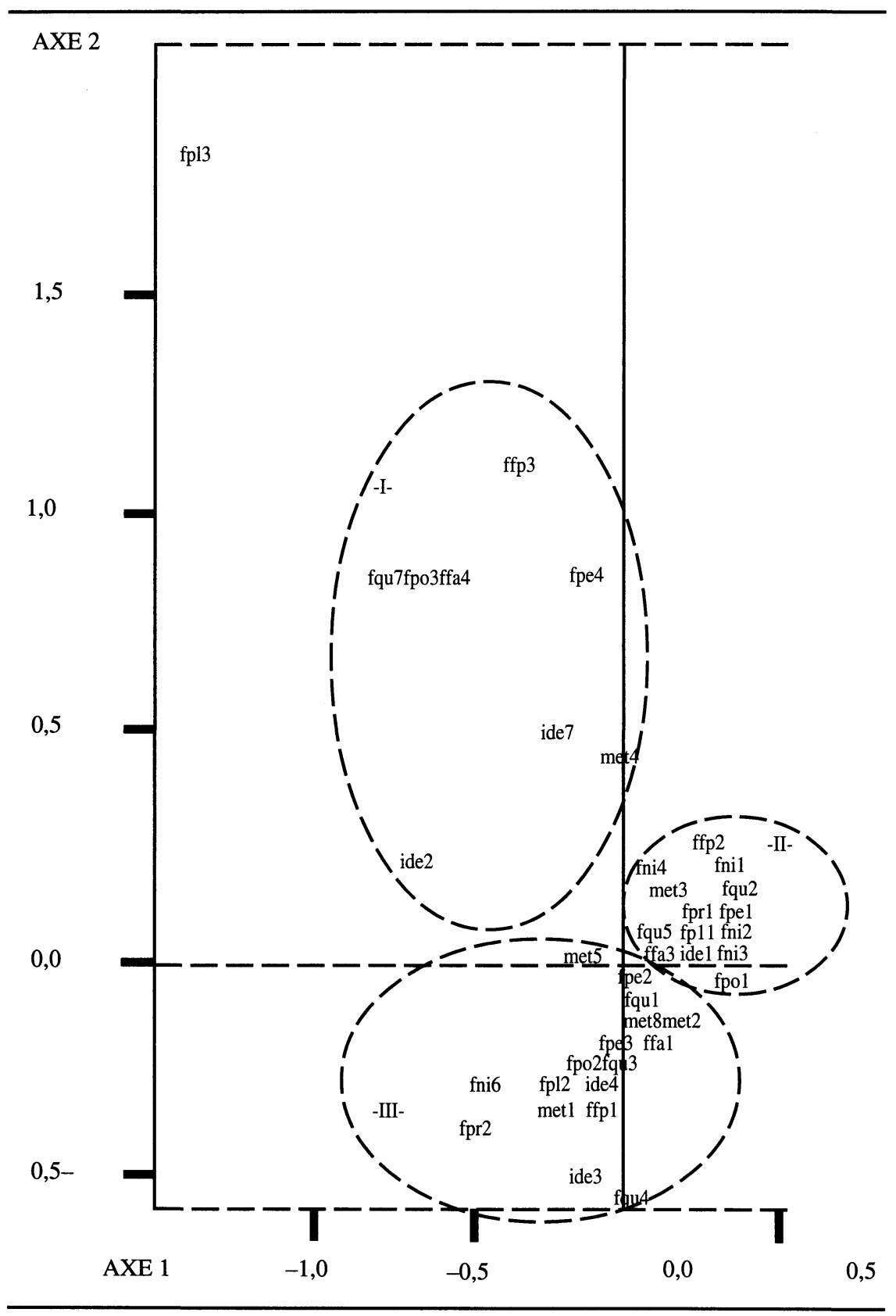


plus d'un an et la gestion de la formation incombe au responsable des ressources humaines ou à une association de plusieurs compétences. Pour ces PME, la formation apparaît comme indispensable et la principale méthode d'évaluation demeure l'observation.

La troisième classe, celle des " ponctuels », a une manière différente d'appréhender la formation. Elle adhère à des FAF, auxquels elle délègue plus volontiers la planification des actions et engage plutôt des formations ponctuelles. Les plans et les projets ne font pas partie des moyens de sa politique, et les seuls plans engagement-développement mis en œuvre le sont depuis moins d'un an. La gestion de la formation incombe soit aux responsables de l'entreprise, soit aux directeurs financiers et administratifs, soit, enfin, aux responsables techniques, commerciaux, aux secrétaires de direction ou aux comptables. La formation apparaît comme un moyen d'action intéressant, mais avec certaines réserves. Les méthodes d'évaluation de la formation semblent inexistantes ou très informelles.

Ces différents groupes identifiés, la question reste de savoir si la poursuite de l'une ou l'autre des politiques apparaît comme un vecteur de performance en milieu PME.

\subsection{Politique de formation et performance}

\section{Liaison positive entre formation suivie et performance}

La performance demeure une notion difficile à appréhender. Le schéma décrivant le système de gestion de la PME (Marchesnay, 1992) a permis d'énumérer un certain nombre d'indicateurs concernant les différents pôles du système et leurs interactions. L'objet des questions était de repérer les évolutions des différentes variables choisies.

Après sélection, 26 variables ont été retenues. Elles décrivent des performances très diverses, telles que le nombre de nouveaux clients, la baisse des réclamations, l'amélioration de la qualité des produits, l'amélioration des gains de productivité grâce au travail, l'évolution du climat de l'entreprise et la circulation de l'information.

Il s'agissait donc de savoir si, en fonction des politiques de formation mises en évidence, il restait possible de relier positivement formation et performance.

C'est la projection des variables de performance sur le plan factoriel qui permet d'apprécier ces liaisons entre politique de formation et performance.

Sur le graphique, de nombreux points apparaissent en superposition. Toutefois, la classification hiérarchique produit les partitions suivantes: 
TABLEAU 2

Formation et performance

\begin{tabular}{ll}
\hline Attitudes/Formation & Indications sur la performance \\
\hline Les indifférents & $\begin{array}{l}\text { Projection des modalités «ne sait pas » et « sans réponse ». } \\
\text { Les passionnés }\end{array}$ \\
$\begin{array}{l}\text { Projection de toutes les modalités indiquant une amélioration } \\
\text { de la performance. } \\
\text { Projection de toutes les modalités indiquant une dégradation } \\
\text { ou une stagnation de la performance. }\end{array}$ \\
\hline
\end{tabular}

Les PME qui s'engagent fortement en formation apparaissent également comme celles qui fonctionnent le mieux. Celles qui ne poursuivent qu' une politique ponctuelle et volontiers déléguée paraissent davantage en difficulté.

Cette analyse permet de croire à l'existence d'une liaison positive entre une politique de formation engagée et une meilleure performance. Les PME qui ne suivent pas une politique élaborée de formation ne sont pas non plus celles qui affichent de bonnes performances.

Pourtant, cela ne peut en aucun cas indiquer l'impact d'une formation engagée sur la performance de l'entreprise. D'autres actions menées parallèlement à la formation peuvent tout aussi bien expliquer cette bonne santé des PME. C'est ce que l'analyse suivante permet de vérifier.

\section{Rapport de causalité entre formation engagée et performance}

Deux projections apparaissent nécessaires pour cette seconde analyse.

La première concerne les variables définies a priori comme pouvant avoir une action sur le système de gestion de la PME. La représentation du système de gestion de Marchesnay a permis la mise en place de ces variables. Au nombre de 12, elles concernent, par exemple, les actions publicitaires, les investissements, les changements organisationnels ou la mise en place de nouvelles techniques de gestion.

La projection des modalités correspondantes sur le plan factoriel donne des résultats significatifs. Un premier découpage de l'arbre lors de la classification hiérarchique permet de distinguer deux groupes. L'un réunit les indifférents, sans projection, et l'autre les passionnés et les ponctuels sur lesquels sont projetées toutes les variables d'action prédéterminées. Un découpage plus fin isole les passionnés et projette les autres actions sur les ponctuels.

Finalement, il semble possible de croire à l'existence d'actions dans un groupe en particulier puisque le groupe des passionnés parait moins concerné par les autres actions que celui des ponctuels. 
La première tendance traduit donc une absence de prédominance d'actions parallèles pouvant agir sur le système de gestion des PME ayant une politique de formation engagée. La performance pourrait alors plus spécialement provenir de cette formation.

Pourtant cette démonstration reste incomplète, car, compte tenu de la multiplicité des variables d'action envisageables, toutes ne peuvent être citées de manière exhaustive. Ainsi, la projection d'une nouvelle série de variables permettrait de pallier cette lacune.

La seconde projection permet donc de compléter l'analyse. Les variables concernées comportent différentes modalités reconstituées à partir de réponses spontanées à des questions ouvertes. Ces questions viennent préciser les actions menées dans le but d'améliorer tel ou tel point du système. Les cinq variables les plus significatives, retenues ici, sont les suivantes :

- les actions pour améliorer la position dans l'environnement;

- les actions pour améliorer la stratégie et les activités ;

- les actions pour perfectionner l'organisation;

- les raisons d'amélioration de la polyvalence;

- les raisons de la progression de la gestion des ressources humaines.

Ces différentes questions font le point sur les grands axes du système de gestion. Leur projection sur le plan et les tendances de la classification hiérarchique permettent de préciser si d'autres actions non encore détectées ont pu agir sur le système de gestion des PME étudiées.

TABLEAU 3

Formation et actions parallèles

\begin{tabular}{ll}
\hline Attitudes / Formation & Indications sur la performance \\
\hline Les indifférents & $\begin{array}{l}\text { Aucune projection. } \\
\text { Les passionnés }\end{array}$ \\
$\begin{array}{l}\text { Projection majoritaire des modalités concernant les actions } \\
\text { de formation. } \\
\text { Les ponctuels }\end{array}$ & $\begin{array}{l}\text { Projection majoritaire des modalités indiquant d'autres types } \\
\text { d'actions. }\end{array}$ \\
\hline
\end{tabular}

Cette projection renforce les observations précédentes. La formation paraît davantage utilisée dans les PME les plus performantes, qui sont aussi celles qui ont une réelle politique de formation. La tentation est grande de voir là un rapport de causalité entre existence de formation et performance. Pourtant, une dernière précaution doit être prise. 


\subsection{Caractéristiques des $\mathrm{PME}$ interrogées}

La dernière projection sur le plan factoriel englobe les différentes modalités caractérisant les PME de l'échantillon. Quatre variables ont été choisies :

- l'effectif : l'échantillon a été sélectionné dans une fourchette d'effectifs allant de 10 à 250 employés, le questionnaire permettant d'identifier différentes classes d'effectifs ;

- l'indépendance : seules les PME dépendantes quant à la prise de décision en formation ont été éliminées. Il reste ainsi dans l'échantillon des PME totalement indépendantes, mais aussi des PME rattachées à de plus grandes structures par des marques, concessions, rapports de sous-traitance ou légère filialisation;

- le type d'activité : une question précise l'orientation plutôt productive ou de prestation de services des PME. Les PME des bâtiments et travaux publics ont été isolées de ces catégories pour en constituer une supplémentaire ;

- le niveau d'expertise nécessaire à l'exercice du métier de l'entreprise : des niveaux bas, moyens et élevés ont été dégagés.

\section{TABLEAU 4}

\section{Formation et caractéristiques}

\begin{tabular}{ll}
\hline Attitudes/Formation & Indications sur la performance \\
\hline Les indifférents & $\begin{array}{l}\text { Aucune projection. } \\
\text { Les passionnés }\end{array}$ \\
& $\begin{array}{l}\text { Projection : effectif entre 100 et } 250 \text { salariés, PME non } \\
\text { totalement indépendantes. } \\
\text { Les ponctuels }\end{array}$ \\
\hline
\end{tabular}

Les variables concernant le niveau d'expertise et le domaine d'activité ne sont pas déterminantes ici. Par contre, la taille joue un rôle très important : les entreprises dont l'effectif est compris entre 100 et 250 salariés (et non totalement indépendantes) s'avèrent les plus performantes. Ce sont aussi celles dont la politique de formation paraît la plus engagée et la plus rigoureuse.

Toutefois, une précision s'impose : l'échantillon des PME de 100 à 250 salariés non totalement indépendantes ne dépasse pas $20 \%$ de l'échantillon total. D'autres PME semblent donc concernées.

L'autre groupe d'entreprises déterminé par une politique de formation plus ponctuelle et plus facilement déléguée semble davantage composé de plus petites organisations totalement indépendantes. Les autres variables projetées ne permettent pas de tirer des conclusions distinctives. 
Le dernier groupe, enfin, ne semble pouvoir être caractérisé par aucune modalité particulière.

La consultation des profils, après vérification de l'existence de liaisons significatives entre les variables d'après le calcul du khi carré, permet de confirmer ces tendances. Ces croisements entre variables de formation et variables caractérisant les PME impliquent plusieurs autres observations :

- le nombre d'entreprises ayant un plan de formation ou affirmant verser plus que le pourcentage obligatoire croît avec la taille de ces PME;

- la formalisation s'accroît ainsi avec la taille de l'entreprise, ce qui a déjà été observé par plusieurs auteurs (Mahé de Boislandelle, 1988; D’Amboise et Bakanibona, 1990 ; Julien, 1993);

- les entreprises totalement indépendantes ont moins souvent un plan de formation et versent plus rarement une somme supérieure au pourcentage obligatoire pour la formation;

- dans les plus petites entreprises, les gestionnaires de la formation semblent plus souvent être les responsables de l'entreprise. Les plus grandes PME, par contre, ont plus souvent un responsable des ressources humaines, ce qui semble confirmé dans diverses études (par exemple, Mahé de Boislandelle, 1988), mais ces PME restent encore nombreuses à laisser à d'autres (chef d'entreprise, responsable financier, etc.) la direction de la formation. Le rôle des chefs de service, techniques ou commerciaux disparaît au-delà de 100 salariés. Les secrétaires de direction, juristes ou comptables n'assurent cette fonction que dans les PME comprenant entre 50 et 150 salariés. Enfin, plusieurs gestionnaires interviennent conjointement à partir de 100 salariés.

Ces différentes observations imposent une limite à l'affirmation de l'influence directe de la formation sur le système de gestion qui, néanmoins, ne vient pas annuler les observations précédentes.

\section{Conclusion}

Suivant les différents résultats, il paraît possible d'affirmer qu'il existe effectivement différentes politiques de formation et que des relations positives entre formation et performance existent. Les deux premières questions qui se posaient à l'origine du travail trouvent donc une réponse affirmative. Il faut ainsi retenir que des politiques de formation ponctuelles, où la formation reste le plus souvent déléguée et finalement peu intégrée aux problèmes concrets de l'entreprise, sont également liées à des performances négatives. 
Tout compte fait, la difficulté réside réellement dans la définition du sens du rapport de causalité, lorsqu'on observe les PME les plus performantes, qui ont une politique de formation engagée et qui sont plus grandes et moins indépendantes que les autres.

SCHÉMA 3

Sens de la causalité

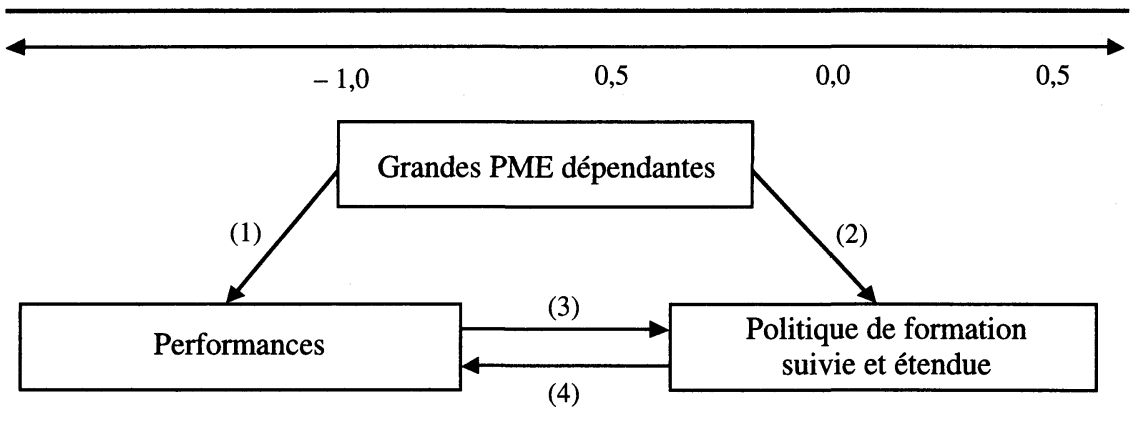

Quatre relations apparaissent.

\section{Le fait d'être une grande PME, non totalement indépendante, permet d'être plus performante.}

Cette première affirmation parait excessive, bien que l'existence d'un biais puisse être envisagée, lié par exemple à une situation particulière des entreprises de 10 à 100 salariés dans la région.

\section{Dans les grandes PME, non totalement indépendantes, la politique de formation semble mieux suivie et plus rigoureuse.}

Cela paraît déjà plus acceptable. Pour diverses raisons, dans la plus petite entreprise, les responsables ne consacrent pas beaucoup de temps à la formation, surtout lorsque celle-ci ne revêt pas un aspect vital ou incontournable. La pression du quotidien leur fait préférer une gestion de la formation au « coup par coup » répondant aux attaques de l'environnement interne ou externe. Ces entreprises restent d'ailleurs plus nombreuses à affirmer être davantage réactives que proactives face aux événements. Elles laissent volontiers la charge de la gestion de la formation à des organismes extérieurs, lesquels leur permettent de mutualiser les fonds. Par contre, dès lors que l'entreprise se structure, que de nouvelles fonctions apparaissent et que le responsable délègue plus volontiers certaines tâches, une politique de formation suivie, engagée, commence à apparaitre. 


\section{La performance conduit les PME à avoir une politique de formation plus suivie et rigoureuse.}

Les moyens apportés par une plus grande performance peuvent en effet permettre à la PME de gérer avec plus de rigueur certaines des fonctions importantes de sa structure. Plus de performance facilite le changement et le progrès dans l'entreprise. Ces derniers impliquent souvent plus de mobilisation en formation et une certaine révélation de l'importance de cette action.

\section{Une politique de formation suivie et rigoureuse améliore la performance.}

Comme les mentions précédentes, celle-ci considérée de manière isolée peut paraitre abusive. Pourtant, la formation apparaît quelquefois comme la variable essentielle de survie et de développement de certaines PME. Cette variable, associée à de nombreux événements de la vie de l'entreprise, prend une dimension d'autant plus grande que la ressource humaine constitue un avantage certain pour la majorité des petites organisations.

Une combinaison de ces différentes assertions permet certainement de mieux approcher la réalité de la PME. L'hétérogénéité du champ laisse penser que les différents cas de figure peuvent se présenter et se combiner pour donner des possibilités variées de rapports de causalité entre les variables étudiées.

Tout un champ de réflexion s'ouvre donc à la recherche dans ce domaine. Il apparaît ainsi, malgré certaines limites du travail, que la réflexion puisse s'engager. Ces limites peuvent être attribuées, par exemple, au contexte même de l'entrevue, laquelle, portant plutôt sur la formation (indication présente une fois dans la lettre d'introduction) risquait d'influencer le répondant. Toutefois, cette influence reste limitée, la première partie du questionnaire traitant du diagnostic de performance, avant les questions sur la formation. Un autre biais envisageable reste la pratique de la formation dans toutes les PME interrogées. Faire de la formation en PME apparaît déjà, en France, comme une démarche volontaire, puisque selon la majorité des études engagées, les PME ne dépensent leurs fonds pour la formation que dans la moitié des cas (De Vendeuil, 1992; AGEFOS-PME, 1992).

L'évaluation de la formation au quatrième niveau, soit celui de la mesure des résultats sur le système de gestion, semble difficile à appréhender et requiert une réflexion à la fois générale et particulière. Générale, car la plupart des indicateurs élaborés dans le questionnaire sont adaptables aux entreprises, dans le cadre de formations classiques. Particulière, car chaque PME doit adapter la réflexion à son cas, en fonction des types de formations suivies, des enjeux de ces actions et du contexte dans lequel elles sont mises en œuvre. 
Quelquefois, comme l'affirme avec sagesse Kirkpatrick (1977), l'évidence peut suffire. En effet, il semble utile de différencier une prise de conscience de la nécessité d'un suivi de la formation et une approche explicite du processus, d'une évaluation structurée, complexe et coûteuse, peu à sa place dans des petites structures manquant de moyens. Ainsi, une réflexion engagée sur les objectifs et leur réalisation dans les PME reste préférable à une méthodologie trop lourde.

Les réflexions et schémas proposés dans cet article donnent des pistes de travail qui doivent être adaptées cas par cas en fonction des objectifs et des contraintes de chaque organisation.

\section{Bibliographie}

AFNOR (1992), Formation professionnelle, Terminologie, Normalisation française X50.750, avril.

AGEFOS-PME (1992), «Perspectives 1993. Les PME face aux ressources humaines et à la formation », Document AGEFOS-PME, 52 p.

BALDWIN, J. (1994), «Stratégies de réussite, profil des PME en croissance au Canada », Statistiques Canada, Ministère de l'Industrie, des Sciences et de la Technologie, p. 59.

BECCARELLI, C. et H. PERKER (1991), « PME-PMI : Définitions et impact socio-économique », Actualité de la Formation permanente, $\mathrm{n}^{\mathrm{o}} 114, \mathrm{p} .37$.

BEL, M. et A. RosanVALLON (1991), «Les politiques innovantes : quelles dynamiques? », Actualité de la Formation permanente, $\mathrm{n}^{\circ} 114$, p. 53-57.

CARON, J. (1994), «Formation continue et entreprise dans le nouveau contexte économique », Québec, Secrétariat du Conseil de la Science et de la Technologie, novembre.

CASPAR, P. et C. Afriat (1988), L'investissement intellectuel, Paris, Economica, p. 40-50.

CEREQ (1992), «Bulletin de recherche sur l'emploi et la formation», Bref, $\mathrm{n}^{\circ} 77$, juin.

D'AмвоISE, G. (1994), «La mise en œuvre des plans et des stratégies», Document de travail 94-19, GREPME, p. 26.

D'AMBOISE, G. et BAKANIBONA (1990), «La planification dans les PME», Revue internationale $P M E$, vol. $3, \mathrm{n}^{\circ}$ 2, p. 148-165.

DEJEAN, P. et J.P. WILLEMS (1991), « De la gestion des sommes à la gestion des hommes », Actualité de la Formation permanente, $\mathrm{n}^{\circ} 114$, p. 69-72.

DE VENDEUIL, R. (1992), «À qui profite la formation continue ?», L'Express, vol. 17, $\mathrm{n}^{\circ}$ 9, p. 4-10.

DIXON, N.M. (1987), «Meet training goals without reaction forms », Personnel Journal, août, p. 108-115.

FAIRFIELD-SONN, J.W. (1987), «A strategic process model for small business training and development », Journal of Small Business Management, janvier, p. 11. 
JULIEN, P.A. (1993), « Vers un modèle général des PME », dans GREPME, Les PME, bilan et perspectives, Ouvrage collectif, Paris, Economica.

KIRKPATRICK, D.L. (1976), «Evaluation of training», dans R.L. Craig, Training and Development Handbook, $2^{\mathrm{e}}$ édition, New York, McGraw-Hill.

KIRKPATRICK, D.L. (1977), «Evaluating training programs : evidence vs proof », Training and Development Journal, novembre.

MCEvoY, G.M. (1983), «Personnel practices in smaller firms : a survey and recommendations », American Journal of Small Business, vol. 8, nº 2, octobre-décembre, p. 36.

MAHÉ de Boislandelle, H. (1988), Gestion des ressources humaines dans les PME, Paris, Economica, $322 \mathrm{p}$.

MAHÉ de BoISLANDELLE, H. (1994), «Esquisse d'une théorisation de la GRH en PME », dans Les Actes du $5^{e}$ Congrès de l'AGRH, Montpellier, 17 et 18 novembre, p. 259-269.

MAHER, J.H. et C.E. KUR (1983), «Constructing good questionnaires », Training and Development Journal, juin.

MARCHESNAY, M. (1992), «Le management stratégique de la PME », Travaux et documents de recherche de l'ERFI, juillet, Montpellier.

MARTORY, B. (1992), Les tableaux de bord sociaux, pilotage, animation, décision, chapitre 5, Paris, Nathan.

MiNTZBERG, H. (1990), Le management, voyage au centre des organisations, Paris, Les Éditions d'Organisation.

ROGER, A. (1985), «Le suivi et l'appréciation du personnel dans les PME de service », Document de travail, IAE Aix-en-Provence, $312 \mathrm{p}$.

TACHE, A. et P. DÉJEAN (1991), «Actualité des représentations de la formation continue et des outils multi-médias : les PME-PMI de la région Midi-Pyrénées », CRIDF, Université des Sciences sociales de Toulouse.

VAN AuKen, P.M. (1982), «La fréquence de la planification stratégique dans les PME », Journal of Small Business Management, juillet.

WALTHER, R. (1988), «Former pour investir », Éducation Permanente, $\mathrm{n}^{\circ}$ 95, décembre, p. 51-62. 